\title{
High-Resolution Spectroscopy of Quasars and Quasar Absorption-Line Systems
}

\author{
J. MICHAEL SHULL ${ }^{1}$ \\ Joint Institute for Laboratory Astrophysics. University of Colorado and National Institute for Standards and Technology. Campus \\ Box 410 , Boulder. Colorado 80309 \\ Electronic mail: mshull casa.colorado.edu \\ Received 1995 March 2l; accepled 1995 June 14
}

\begin{abstract}
I provide a summary of the session on "Active Galactic Nuclei and Quasar Absorption Lines," with contributions by J. Wampler, A. Wolfe, W. Sargent, J. Bechtold, M. Pettini, and M. Shull. The topics included new observations of QSO absorption lines by the Keck Telescope HIRES spectrometer and the Hubble Space Telescope. An overview of the major scientific issues in this field is followed by a brief summary of a panel discussion that addressed future instrumental possibilities that could answer some of these questions.
\end{abstract}

\section{OVERVIEW: WHY IS HIGH RESOLUTION NEEDED?}

Because the spectra of quasars and other active galactic nuclei ( $A G N$ ) are dominated by broad emission lines, one might regard these objects as unlikely "scientific drivers" for high-resolution spectroscopy. In fact, the narrow absorption lines from $\mathrm{H} I$ and heavy elements seen toward AGN provide some of the most powerful incentives for observations with the largest telescopes using spectrographs with resolutions of $30,000-100,000$. Many of these absorption systems are believed to arise from the disks, halos, and collisional debris of interrening galaxies (Bahcall and Spitzer 1969; Weymann et al. 1981). However, a class of absorbers known as the "Lyman $\alpha$ forest. " because of their high abundance shortward of the $1216 \AA$ Ly $\alpha$ emission line, is believed to represent a population of true intergalactic clouds (Sargent et al. 1980; Weymann 1993).

Expressed in velocity units, the broad emission lines in AGN are typically $10,000 \mathrm{~km} \mathrm{~s}^{-1}$ wide and the narrow emission lines are $\leqslant 1000 \mathrm{~km} \mathrm{~s}^{-1}$ wide, while the metal absorption lines have typical widths of $10-30 \mathrm{~km} \mathrm{~s}^{-1}$ or less. Recent surveys of Ly $\alpha$ forest clouds have found mean or median doppler widths ( $b$ values) ranging between 30 and 35 $\mathrm{km} \mathrm{s}^{-1}$ (Rauch et al. 1992, 1993). For reference, thermal broadening of hydrogen lines at temperature $T=\left(10^{4} \mathrm{~K}\right) T_{4}$ produces a Doppler width $b=\left(2 \mathrm{kT} / \mathrm{m}_{\mathrm{H}}\right)^{1 / 2}=(12.9 \mathrm{~km}$ $\left.\mathrm{s}^{-1}\right) T_{+}^{1 / 2}$. One study at $6-7 \mathrm{~km} \mathrm{~s}^{-1}$ resolution (Pettini et al. $1990)$ suggested that some $b$ values could be as low as 10 $\mathrm{km} \mathrm{s}^{-1}$. The latter observations were surprising, since thermal equilibrium of low-density. low-metallicity gas photoionized by quasar radiation should result in temperatures in excess of $30.000 \mathrm{~K}$ (Donahue and Shull 1991). Recent data from the Keck Telescope (Tytler 1994) show that most of the $\mathrm{HI}$ (Ly $\alpha$ forest) lines have $22<b<36 \mathrm{~km} \mathrm{~s}^{-1}$, but some may be nartower. In view of the nonequilibrium flows found in recent numerical hydrodynamic models of baryonic infall in the Cold Dark Matter (CDM) or Mixed Dark Matter (MDil) paradigm (Cen and Ostriker 1994; Katz et al. 1995), these issues have gained renewed imporance.

'Also ai Center for Astrophysics and Space Astronomy. Deparment of Astrophusical. Planetary. and Aimospheric Physics.
Line profile studies of the metal-line and Ly $\alpha$ forest clouds require $3-6 \mathrm{~km} \mathrm{~s}^{-1}$ resolution (at good signal to noise) to distinguish line asymmetries and to search for substructure. Reliable studies of thermal widths require similar resolution, particularly to separate blends and settle the debate over low $b$ values. Studies of nuclear outflows can be done at $50-100 \mathrm{~km} \mathrm{~s}^{-1}$ resolution. as seen in the phenomena of "associated absorbers" or "satellite emission lines" (U)rich et al. 1985; Clavel et al. 1991; Stocke et al. 1994).

\section{MAJOR ISSLES}

In this section, I describe some of the current scientific issues in the field of quasar absorption lines. This discussion is brief, and readers interested in more extensive information should consult review papers from recent conferences, such as the 1988 STScI Symposium (Blades et al. 1988), the 1992 Tetons Meeting (Shull and Thronson 1993), and the 1994 Munich (ESO) Conference (proceedings in press, 1995).

\subsection{Origin and Physical State}

Historically, Ly $\alpha$ absorption lines have been subdivided into three classes, depending on their column density, $N(\mathrm{H} \mathrm{I})$ $\left(\mathrm{cm}^{-2}\right)$. With some latitude in the definitions, these classes are: (1) the Ly $\alpha$ forest clouds $\left[12.5<\log N^{\prime}(\mathrm{H} \mathrm{H})<17.0\right]$ : (2) the Lyman Limit (LL) systems $[16.5<\log N(H \mathrm{I})<20]$ : and the damped Ly $\alpha$ systems $[20.3<\log N(\mathrm{H} \mathrm{I})<21.8]$. The Ly $\alpha$ absorbers have been observed to have Doppler widths ranging from $b=10-50 \mathrm{~km} \mathrm{~s}^{-1}$. With a mean around $30 \mathrm{~km} \mathrm{~s}^{-1}$. The Ly $\alpha$ line-center optical depth is given by $\tau_{0}$ $=(0.253) N_{13} b_{30}^{-1}$. where $N_{13}$ is the $\mathrm{HI}$ column density in units $10^{13} \mathrm{~cm}^{-i}$ and $b_{30}$ is the Doppler width in units 30 $\mathrm{km} \mathrm{s}^{-1}$. For systems with $\log N(\mathrm{H} \mathrm{l}) \geqslant 1+.5$. it becomes increasingly difficult to infer an accurate $\mathrm{H}$ I column density. owing to saturation (curve-of-growth) problems. In fact, the existence of a continuous power-law distribution ranging from $10^{12.9}$ up to $10^{17} \mathrm{~cm}^{-?}$ in the Ly $\alpha$ forest is not at all certain because of this problem. Some authors (Kulkarni and York 1993; Meiksin and Madau 1993) have suggested a cutoff in the numbers of systems with $N(\mathrm{H} \mathrm{I})>10^{1+5} \mathrm{~cm}^{-2}$. The situation should soon be clarified by studies of the higher 
implemented, one should probably regard the simulated $\mathrm{H}$ I distributions with some skepticism. Nevertheless, the simulations have changed the picture of the possible geometries of the Ly $\alpha$ absorbers and they illustrate the complexities that might be expected to arise from shocks, velocity-space "caustics," and nonequilibrium effects of bulk motions on the gas densities and temperatures.

Related questions also arise in these scenarios. Are the absorbers associated with galaxies in any way-as extended $\mathrm{H} I$ disks, clouds in halos, companion galaxies, or debris clouds? Are they confined by gravity, static thermal pressure, ram pressure, or all of the above? Do the absorbing clouds retain kinematic signatures from these processes? Highresolution spectra, capable of studying line profiles at better than $10 \mathrm{~km} \mathrm{~s}^{-1}$, could address these issues.

\subsection{Evolution of Metallicity}

One of the most intriguing recent scientific results in this field is the detection of trace heavy elements in high-columndensity ("damped") Ly $\alpha$ absorbers (Meyer et al. 1989; Pettini et al. 1994; Wolfe et al. 1994). These elements most likely came from stars, but of what type and at what redshift? Determining the evolution rate of metallicity as a function of redshift, $Z(z)$, is a critical spectroscopic problem for large telescopes. The key elements are: $\mathrm{C}, \mathrm{N}, \mathrm{O}, \mathrm{Si}, \mathrm{Mn}, \mathrm{Mg}, \mathrm{Fe}$, and trace species such as $\mathrm{Zn}, \mathrm{Ni}, \mathrm{Cr}, \mathrm{Co}$, and $\mathrm{Ti}$. Accompanying the study of the overall metallicity history is the problem of estimating the degree to which massive stars have contributed to the production of hot gas and ionizing (Lyman continuum) photons. Until recently, most astronomers have interpreted the absorption lines from Lyman-limit systems and damped Ly $\alpha$ systems as gas photoionized by a powerlaw (hard) radiation field from quasars. The possibility of supernovae enrichment and hot-star contributions to the local radiation field (Giroux et al. 1994; Sutherland and Shull 1995) makes the interpretation far richer scientifically. From current models of massive-star evolutionary tracks (Schaller et al. 1992), stellar atmospheres (Kurucz 1992), and heavyelement yields (Woosley and Weaver 1995), it now appears that a population of massive stars $\left(8-85 M_{\odot}\right)$ is capable of producing significant amounts of oxygen, hot gas, and Lyman continuum ( $\mathrm{LyC}$ ) photons. Quantitatively, the efficiency of LyC energy production, normalized to metal production, is given by the dimensionless parameter (Madau and Shull 1995),

$$
\eta_{\text {hyc }}=\frac{\int_{\nu_{0}}^{x} L_{\nu} d \nu}{M_{m} c^{2}} \approx 0.002,
$$

where $M_{m}$ is the mass of metals produced and $L_{v}$ is the spectral luminosity (ergs s${ }^{-1} \mathrm{~Hz}^{-1}$ ) of radiation in the LyC $\left(\nu \geqslant \nu_{0}\right)$. If the metals are produced steadily, from redshift $z \approx 5$ to the present, the ionizing photons from hot stars could rival the background from $A G N$.

\subsection{Reionization of the Intergalactic Medium}

Because of the observed absence of the HI GunnPeterson effect back to redshifts $z \approx 4$, the IGM must have been highly ionized by this epoch, presumably by quasars and perhaps also by hot stars. As noted earlier, the He II Gunn-Peterson effect (Jakobsen et al. 1994; Tytler 1994) may be caused by line blanketing by the Ly $\alpha$ forest if clouds are present down to columns $N(\mathrm{H} \mathrm{I})=10^{12} \mathrm{~cm}^{-2}$ and if the ionizing radiation field is sufficiently soft above 4 Ry to allow He IV/H I>100 (Madau and Meiksin 1994; Giroux et al. 1995). A portion of the required softness (4 to 1 Ry ratio) could be provided by the "filtering" of quasars" spectra by the photoelectric absorption in intervening Ly $\alpha$ clouds. Detailed models, however, suggest that the sources still must have spectral indices $\alpha_{s}>1.4$, somewhat steeper than the canonical $\nu^{-1}$ AGN spectrum. An important set of future issues in this area involve understanding the relative roles of quasars and starburst galaxies in producing this radiation field.

\section{PANEL DISCUSSION}

The final portion of this session consisted of a panel discussion with audience participation. The panelists were Jill Bechtold, Wal Sargent, Joe Wampler, Max Pettini. and Art Wolfe, with Mike Shull as moderator. After the panel's comments, the discussion was opened to comments from instrumentalists and the audience. Five general questions were asked of the panel:

(1) What spectral resolution is needed?

(2) What signal-to-noise ratio is needed?

(3) What is the need for high resolution in the infrared?

(4) What further progress can be made on $\mathrm{D} / \mathrm{H}$ from the ground?

(5) What is your "wish list" for new instruments and technology?

Comments by Wampler: In his presentation, Joe Wampler described evidence for $\mathrm{Mg}$ II and $\mathrm{Al} \mathrm{III} \mathrm{"waves"} \mathrm{in}$ broad absorption line (BAL) quasar spectra. perhaps indicative of a line-locking mechanism. Studies of these features require extremely high signal-to-noise spectra at high resolution $(R>100,000)$.

Comments by Wolfe: Ar Wolfe discussed the results of a survey of 570 QSOs containing 61 damped Lya systems in which $\mathrm{Zn}, \mathrm{Cr}, \mathrm{Co}, \mathrm{Ni}$, and $\mathrm{Fe}$ absorption lines were analyzed. The first major result was that, at high redshift $(z \approx 3-4)$, the amount of baryonic matter in these systems approaches that attributable to luminous galaxies today $\left(\Omega_{\text {damp }} \approx \Omega_{*} \approx 0.005\right)$. A second major result, from the Keck Telescope, was a determination (Wolfe et al. 1994) of accurate abundances for trace metals in a damped absorber at $z=2.3$ toward PHL 957. At $8 \mathrm{~km} \mathrm{~s}^{-1}$ resolution, these data showed $[\mathrm{Zn} / \mathrm{H}]$ $=-1.55 \pm 0.11,[\mathrm{Cr} / \mathrm{H}]=-1.79 \pm 0.10$, and $[\mathrm{Ni} / \mathrm{H}]=-2.13$ \pm 0.18 , with an upper limit on $[\mathrm{O} / \mathrm{H}]<-0.97$. A key use for high resolution comes from the need to separate several lines blends ( $\mathrm{Zn} \mathrm{II,} \mathrm{Cr}$ II, Co II, Mg I) from these features. The kinematics of these metal systems suggest vertically thick disks with rotational velocities $V_{\text {rot }} \approx 200 \mathrm{~km} \mathrm{~s}^{-1}$ over scale lengths $5-10 \mathrm{kpc}$.

Comments by Sargent: Wal Sargent presented a number of Keck HIRES spectra of absorption systems, including $\mathrm{Na} I$ $D$ lines in a tidal tail of a galaxy $(z=0.0518)$ in front of a 
Madau, P., and Meiksin, A. 1994, ApJ, 433, L53

Madau, P., and Shull, J. M. 1995, ApJ (in press)

Meiksin, A., and Madau, P. 1993, Apl, 412, 34

Meyer, D. M., Welty, D. E., and York, D. G. 1989, ApJ, 343, L37

Miralda-Escudé, J., and Ostriker, J. P. 1990, ApJ, 350, 1

Moller, P., and Jakobsen, P. 1990, A\&A, 228, 299

Murdoch, H. S., Hunstead, R. W., Pettini, M., and Blades, J. C. 1986, 309, 19

Pettini, M., Hunstead, R. F., Smith, L. J., and Mar, D. P. 1990 , MNRAS, 246, 545

Pettini, M., Smith, L. J., Hunstead, R. W., and King, D. L. 1994, ApJ, 426, 79

Press, W. H., and Rybicki, G. B. 1993, ApJ, 418, 585

Rauch, M., Carswell, R. F., Chaffee, F. H., Foltz, C. B., Webb, J. K., Weymann, R. J., Bechtold, J., and Green, R. F. 1992. ApJ, 390, 387

Rauch, M., Carswell, R. F., Webb, J. K., and Weymann, R. J. 1993, MNRAS, 260, 589

Sargent, W. L. W., Young. P. J., Boksenberg. A., and Tytler, D. 1980, ApJS, 42, 41

Schaller, G., Schaerer, D., Meynet, G., and Maeder, A. 1992, A\&AS, 96, 269

Shull, J. M., and Thronson, H. A. (eds.) 1993, The Environment and Evolution of Galaxies (Dordrecht, Kluwer)
Songaila, A., Cowie, L. L., Hogan, C. J., and Rugers, M. 1994, Nature, 368, 599

Songaila, A., Hu, E. M., and Cowie, L. L. 1995. Nature, 375, 124 Steidel, C. C. 1993, in The Environment and Evolution of Galaxies, Proceedings of Third Tetons Conference, ed. J. M. Shull and H. A. Thronson (Dordrecht, Kluwer), p. 263

Steidel, C. C., and Sargent, W. L. W. 1987, ApJ, 318, L11

Stocke, J., Shull, J. M., Granados, A., and Sachs, E. R. 1994, AJ, 108,1178

Sutherland, R., and Shull, J. M. 1995, ApJ (submitted)

Tytler, D. 1994, invited paper at Munich Conference (ESO)

Ulrich, M.-H., et al. 1985, Nature, 313, 745

Weymann, R. J. 1993, in The Environment and Evolution of Galaxies, Proceedings of Third Tetons Conference, ed. J. M. Shull and $H$. A. Thronson (Dordrecht, Kluwer), p. 213

Weymann, R. J., Carswell, R. F., and Smith, M. G. 1981, ARAA, 19. 41

Wolfe, A. M. 1990, in The Interstellar Medium in Galaxies, ed. H. A. Thronson and J. M. Shull (Dordrecht, Kluwer), p. 387

Wolfe, A. M., Fan, X.-M., Tytler, D., Vogt, S. S., Keane, M. J., and Lanzetta, K. M. 1994, ApJ, 435, L101

Woosley, S., and Weaver, T. 1995, ApJS, 101 (in press)

Zheng, W., and Davidsen, A. 1995, ApJ, 440, L53 
